\title{
A Game Theoretic Framework for joint Routing and Pricing in Networks with Elastic Demands .
}

\author{
Eitan Altman \\ INRIA Sophia Antipolis \\ 2004 Route des Lucioles \\ B.P. 93, 06902 Sophia \\ Antipolis Cedex, France \\ Eitan.Altman@sophia.inria.fr
}

\author{
Jocelyne Elias \\ Department of Electronics and \\ Information \\ Politecnico di Milano \\ $34 / 5$ Via Ponzio \\ Milano 20133, Italy \\ elias@elet.polimi.it
}

\author{
Fabio Martignon \\ Department of \\ Information Technology \\ and Mathematical Methods \\ University of Bergamo \\ Dalmine (BG) 24044, Italy \\ fabio.martignon@unibg.it
}

\begin{abstract}
In this paper, we study the economic interactions between network users and providers. Each user must ship his traffic from a source to a destination node, splitting it over multiple paths, each owned by an independent network provider. Users are charged a fixed price per unit of bandwidth used, and face both access and transport costs. The transmission rate of each user is assumed to be function of network congestion (like for TCP traffic) and the price per bandwidth unit. Network providers compete among themselves to cover network users, and set transport prices to maximize their revenue.
\end{abstract}

We provide sufficient conditions for the existence and the uniqueness of the Nash equilibrium under a variety of cost functions, and we derive optimal price and routing settings. Finally, we analyze and discuss several numerical examples that provide insights into the models' solution.

\section{Keywords}

Routing, Pricing, Stackelberg Game, Elastic Traffic

\section{INTRODUCTION}

The complexity of modern, large-scale networks calls for decentralized control algorithms, where routing, pricing, network design and control decisions are made by each network entity independently, according to its own individual performance objectives.

Permission to make digital or hard copies of all or part of this work for personal or classroom use is granted without fee provided that copies are not made or distributed for profit or commercial advantage and that copies bear this notice and the full citation on the first page. To copy otherwise, to republish, to post on servers or to redistribute to lists, requires prior specific permission and/or a fee.

VALUETOOLS 2009, October 20-22, Pisa, Italy

Copyright (C) 2009 ICST 978-963-9799-70-7

DOI 10.4108/ICST.VALUETOOLS2009.7512
The design and operation of such networks is not handled by a central authority, but arises from the interactions of a high number of self-interested agents. This is the case of the Internet, where connectivity is ensured by Autonomous Systems' agreements, but also of overlay networks built on top of the Internet, where a large number of independent Service Providers seek to selfishly optimize the quality and cost of their own network, while covering the largest set of customers, to increase their revenue.

These networks are henceforth called noncooperative, and game theory provides the systematic framework to study and understand their behavior.

Competitive Routing with selfish users in the context of telecommunication networks has been the focus of several works $[1,2,3]$. Noncooperative games in the context of competitive routing and pricing were initially studied in the area of transportation networks $[4,5,6,7]$. Nonetheless, the user considered in such networks controls just an infinitesimally small portion of the network flow, whereas, in this work, we are concerned with users that control non-negligible portions of flow. The joint problem of routing and price setting is also tackled in $[5,6,8,9]$, making at least one of the following limiting assumptions: (1) infinitesimally small users are considered and (2) a monopolist service provider manages the whole network.

In this paper, we overcome these limitations by proposing a novel game theoretic model that solves the joint problem of noncooperative routing and price setting considering both multiple Service Providers (SPs), which set prices for their links in the network, and a given set of users which are characterized by elastic traffic demands that must be routed over one or multiple links.

We model the interaction between the SPs and the users as a Stackelberg game [10]. SPs set their prices and the users respond by presenting a certain amount of flow to the network. The users do not cooperate among themselves, thus leading to a Nash game.

Then, we extend such model taking into account the access costs incurred by the users to ship their flows on the transit links through a set of access nodes. 
We study analytically the existence and uniqueness of the Nash Equilibrium Point (NEP) for our Stackelberg game. Next, we obtain explicit expressions for the users' flows and the optimal prices along with the conditions under which the NEP exits and is unique.

Finally, we analyze and discuss several numerical examples that illustrate the following results: first, the optimal price that service providers should impose is independent from the number of users, and it is proportional to the average utility per traffic unit experienced by network users. Second, as the number of users grows, the revenue achieved by network providers increases until it reaches a saturation point. Finally, the service provider's revenue is very sensitive to access costs, especially when the users number is small.

In summary, this paper makes the following main contributions:

- a novel game theoretical framework for the joint routing and pricing problem considering both multiple SPs and users with elastic traffic demands.

- An extended model that takes into account the access costs incurred by network users to ship their flows on the SPs' links through a set of access nodes.

- An exhaustive study of the existence and uniqueness of the Nash equilibrium and the determination of the conditions under which the Nash equilibrium exists and is unique.

- A thorough analysis of different numerical examples that provide interesting insights into the models' solution.

We underline that our proposed joint routing and price setting framework (and the specific results obtained) would be of particular interest in several network scenarios, including for example overlay $[11,12,13]$ and MPLS networks [14].

A first important application is represented by Service Overlay Networks (SONs), which have recently emerged as an alternative and very promising architecture able to provide end-to-end Quality of Service guarantees in the Internet, while leaving the underlying Internet infrastructure unchanged $[11,12,13]$. An overlay network is an application-layer network built on top of the traditional IP-layer networks, and consists of overlay nodes residing in the underlying ISP domains. Overlay nodes can perform service-specific data forwarding and control functions, and are interconnected by virtual overlay links which correspond to one or more IPlayer links [11]. The service overlay architecture is based on business relationships between the SON, the underlying ISPs, and the users. The SON establishes bilateral service level agreements with the individual underlying ISPs to install overlay nodes and purchase the bandwidth needed for serving its users. On the other hand, the users subscribe to SON services, which will be guaranteed regardless of how many IP domains are crossed by the users' connection. The SON gains from users' subscriptions.

On the other hand, in the MPLS architecture the traffic needs to be partitioned into traffic trunks, or equivalently, the incoming traffic needs to be split over a set of links, and routing decisions are implemented locally by each source, typically in a noncooperative manner.

More in general, the network of parallel links adopted in our work constitutes an appropriate model for several networking problems: (a) modern networking, where resources are pre-allocated to various non-interfering routing paths, (b) broadband networks, where the bandwidth is partitioned among different virtual paths and (c) internetworking, where each link represents a different sub-network.

The paper is structured as follows: Section 2 discusses related work. Section 3 introduces the network model, including users' and SPs' objective functions, as well as cost functions. Section 4 demonstrates the existence and uniqueness of the Nash Equilibrium Point (NEP), while Section 5 computes the NEP along with the conditions under which such equilibrium exists and is unique. Section 6 determines the NEP for the special case of symmetrical users. Section 7 first describes the extended model, which includes users' access costs, and then computes the solutions for such model. Section 8 discusses several numerical examples that illustrate how our model captures interesting aspects of the interaction between network users and providers. Finally, Section 9 concludes this paper.

\section{RELATED WORK}

Several works are proposed in the literature to tackle the joint problem of routing and price setting with one or multiple service providers $[5,6,7,8,9,15]$.

In [5], the authors deal with the routing and pricing problem considering one class of infinitesimally small users, multiple service providers (SPs) and elastic traffic. The authors consider a parallel links network and focus on a single-stage game, where the only players are edge owners who set prices and anticipate the resulting flow.

A similar problem is addressed in [6]. The author computes the equilibrium prices considering affine latency functions: they provide a full characterization of pure strategy oligopoly equilibria and a tight bound on the efficiency of such equilibria.

Another joint routing and pricing problem is addressed in [7], where different classes of infinitesimally small users and one monopolist service provider are considered in a parallel links network scenario.

The same problem is tackled in [15] considering first a monopolist service provider and then multiple service providers that compete among themselves. These SPs play a price competition game and the equilibrium in this case is named the oligopoly equilibrium.

In [8], a Stackelberg game is considered to address the routing and pricing problem with a monopolist service provider and a finite number of users that share the SP link of limited capacity. The leader is the SP, which sets the price, and the followers are the users, which route their flow maximizing their objective function. The user's utility is represented by a logarithmic function, $w_{i} \log \left(1+x_{i}\right)$, where $x_{i}$ is the total 
flow of user $i$. The equilibrium is computed analytically.

The same game is considered in [9] with different classes of finite number of users and a tandem links network. Also in this work, the equilibrium is computed analytically along with the conditions under which the equilibrium exists and is unique.

These two previous works are quite similar to our problem; in our work, however, we consider multiple service providers which set prices for their edges in a parallel links network. Moreover, in [8,9], while defining the objective function, the users' congestion cost is set equal to a simple expression, i.e. $\frac{1}{c_{l}-x_{l}}$, where $c_{l}$ and $x_{l}$ are the capacity of link $l$ and the total flow that traverses link $l$, respectively. On the contrary, in our work, the congestion cost on link $l$ is expressed per unit of flow (see the expression of the $i$-th user objective function, equation (1)), which both produces a more complex problem and provides interesting insights into the problem solution.

\section{NETWORK MODEL}

The adopted model is a network of parallel links illustrated in Figure 1, where a source node $S$ and a destination node $D$ are interconnected by a set of parallel communications links, $\mathcal{L}=\{1, \ldots, L\}$. Each link is owned and managed by a distinct Service Provider (SP).

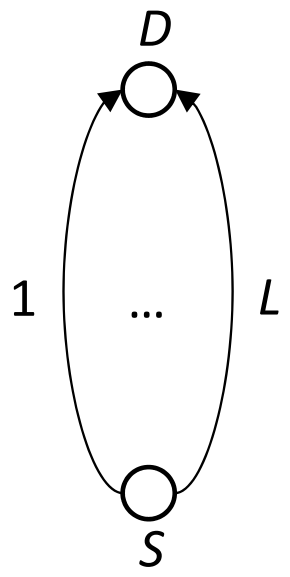

Figure 1: Parallel Links Network: a source node $S$ and a destination node $D$ are interconnected by a set of $L$ parallel communications links, each owned and managed by a distinct Service Provider. A set of $I$ users must ship their flow from $S$ to $D$.

We consider a set $\mathcal{I}=\{1, \ldots, I\}$ of users; each user ships his flow from $S$ to $D$ by splitting his traffic over the set of parallel links, according to some individual performance objective, thus choosing how much of his traffic will be transported by each SP. Users demands are elastic (like for TCP traffic), in the sense that they are function of the prices set by SPs and the costs due to link congestion.

Let $f_{l}^{i}$ denote the expected flow that user $i$ sends on link $l$. The user flow configuration $f^{i}=\left\{f_{1}^{i}, \ldots, f_{L}^{i}\right\}$ is called a routing strategy of user $i$, and the set of strategies $F^{i}=$ $\left\{f^{i} \in R^{L}: f_{l}^{i} \geq 0, l \in \mathcal{L}\right\}$ is called the routing strategy space of user $i$.

The system flow configuration $f=\left\{f^{1}, \ldots, f^{I}\right\}$ is called a routing strategy profile, and takes values in the product strategy space $F$.

Each individual user $i \in \mathcal{I}$ aims at maximizing his degree of satisfaction (his objective function $F^{u, i}$, defined in the following), which we assume has a component related to the throughput (utility) and another one related to costs (disutility).

We assume that a group $I^{S P}=\{1, \ldots, L\}$ of Service Providers $(S P)$ participates in the game. We associate a $\mathrm{SP}$ with each one of the $L$ links. The SPs behave selfishly, and play to maximize their own profit. Each SP $l$ defines its own price strategy $p_{l} \in R$, where $p_{l}$ represents the price per bandwidth unit charged by SP $l$ for all the traffic that passes through link $l$. The collection of the SP strategies builds up the price strategy profile $p=\left(p_{1}, p_{2}, \ldots, p_{L}\right) \in R^{L}$.

The vector $(f, p) \in R^{I L+L}$ grouping both the routing and the price strategy profiles is referred to as network strategy profile and represents the solution of the game where both the individual users and the SPs simultaneously operate.

\subsection{User Objective Function}

We associate to user $i \in \mathcal{I}$ the objective function $F^{u, i}$, which is a function of the flow routed in the network as well as of the prices set by the Service Providers:

$$
\begin{array}{r}
F^{u, i}\left(f^{i}, f^{-i}, p\right)=Q^{i}\left(f^{i}\right)-\left[\sum_{l \in \mathcal{L}} f_{l}^{i} \cdot J_{l}^{i}\left(f^{i}, f^{-i}\right)+\right. \\
\left.+\sum_{l \in \mathcal{L}} f_{l}^{i} \cdot p_{l}\left(f_{l}^{i}, f_{l}^{-i}\right)\right]
\end{array}
$$

The first term, $Q^{i}\left(f^{i}\right)$, represents the utility for routing a total amount of flow $\sum_{l \in \mathcal{L}} f_{l}^{i}=f^{i}$. The second term encompasses two components: the first one, $\sum_{l \in \mathcal{L}} f_{l}^{i} \cdot J_{l}^{i}\left(f^{i}, f^{-i}\right)$, is the disutility due to link delay and the second one, $\sum_{l \in \mathcal{L}} f_{l}^{i}$. $p_{l}\left(f_{l}^{i}, f_{l}^{-i}\right)$ corresponds to the price paid by user $i$ to the Service Providers. Recall that $p_{l}\left(f_{l}^{i}, f_{l}^{-i}\right)$ is the price per bandwidth unit set by the $l-$ th SP.

Hereafter we assume that $Q^{i}\left(f^{i}\right)$ is equal to $\sum_{l \in \mathcal{L}} \alpha_{l}^{i} \cdot f_{l}^{i}$ and $p_{l}\left(f_{l}^{i}, f_{l}^{-i}\right)$ is constant and equal to $p_{l} ; \alpha_{l}^{i}$ therefore represents the utility of user $i$ per unit of transmitted flow, on link $l$. Hence, the objective function of user $i$ becomes as follows:

$$
F^{u, i}\left(f^{i}, f^{-i}, p\right)=\sum_{l \in \mathcal{L}} \alpha_{l}^{i} \cdot f_{l}^{i}-\sum_{l \in \mathcal{L}}\left[f_{l}^{i} \cdot\left(J_{l}^{i}\left(f^{i}, f^{-i}\right)+p_{l}\right)\right] .
$$

Each user $i$ aims to maximize his objective function $F^{u, i}$ over all his flow configurations: 


$$
\max _{f_{l}^{i}}\left\{\sum_{l \in \mathcal{L}} \alpha_{l}^{i} \cdot f_{l}^{i}-\sum_{l \in \mathcal{L}}\left[f_{l}^{i} \cdot\left(J_{l}^{i}\left(f^{i}, f^{-i *}\right)+p_{l}^{*}\right)\right]\right\}
$$

where $p_{l}^{*}$ is the optimal price set by Service Provider $l$ and $f^{-i *}$ are the optimal flows of all users $j \in \mathcal{I}$, with $j \neq i$.

\subsection{Service Provider Utility Function}

The utility function of the $l$-th Service Provider, $U_{l}^{S P}$, is given by:

$$
U_{l}^{S P}\left(f, p^{l}, p^{-l}\right)=p_{l} \cdot f_{l}
$$

where $f_{l}=\sum_{i \in \mathcal{I}} f_{l}^{i}\left(f_{l} \geq 0\right)$ is the total amount of flow on link $l$.

Each SP $l$ aims to maximize its utility $U_{l}^{S P}$ over all its price strategies:

$$
\max _{p_{l}}\left\{p_{l} \cdot f_{l}\right\}
$$

\subsection{Polynomial Link Costs}

In this work, following the guidelines of [3], we consider a class of polynomial link cost functions originally adopted in the context of road traffic modeling [16]. Such costs have appealing properties that lead to predictable and efficient network flows, ensuring the uniqueness of the Nash equilibrium point, as we will establish in the next Section.

More specifically, we assume that each link $l$ is characterized by the following cost function:

$$
\operatorname{cost}(l)=a_{l} \cdot\left(f_{l}\right)^{\beta(l)}+b_{l}, \forall l \in \mathcal{L},
$$

where $a_{l}, b_{l}$ and $\beta(l)$ are link-specific positive parameters, and $f_{l}=\sum_{i \in \mathcal{I}} f_{l}^{i}$ is the total amount of flow that is routed over link $l$.

This is the cost adopted by the US Bureau of Public Roads [16]. The additive term $b_{l}$ here could be interpreted as an additional fixed toll per traffic unit for the use of link $l$.

\section{EXISTENCE AND UNIQUENESS OF THE NASH EQUILIBRIUM POINT (NEP)}

In this Section we demonstrate the existence and uniqueness of the Nash Equilibrium Point (NEP), considering the polynomial cost function (6) defined previously.

We denote by $F_{l}^{u, i}\left(f^{i}, f^{-i}, p\right)$ the user objective function on link $l$, which is equal to:

$$
F_{l}^{u, i}\left(f^{i}, f^{-i}, p\right)=\alpha_{l}^{i} \cdot f_{l}^{i}-\left(f_{l}^{i} \cdot\left[a_{l} \cdot\left(f_{l}\right)^{\beta(l)}+b_{l}+p_{l}\right]\right)
$$

We have therefore the following objective function for each user $i \in \mathcal{I}$ :

$$
F^{u, i}\left(f^{i}, f^{-i}, p\right)=\sum_{l \in \mathcal{L}} \alpha_{l}^{i} \cdot f_{l}^{i}-\sum_{l \in \mathcal{L}} f_{l}^{i} \cdot\left[a_{l} \cdot\left(f_{l}\right)^{\beta(l)}+b_{l}+p_{l}\right] .
$$

\subsection{Existence of the NEP}

The $i$-th user objective function (8) is continuous in $f=$ $\left\{f^{1}, \ldots, f^{I}\right\}$ and concave in $f_{l}^{i}$ (the second partial derivatives of $F^{u, i}(f)$ with respect to $f_{l}^{i}$ are equal to $-a_{l} \cdot \beta(l)$. $\left(f_{l}\right)^{[\beta(l)-2]} \cdot\left[2 \cdot f_{l}+(\beta(l)-1) \cdot f_{l}^{i}\right]$ and are therefore negative $\forall f_{l}^{i} \geq 0, f_{l} \geq 0$ and $\left.\beta(l)>0\right)$. These properties ensure the existence of the Nash equilibrium [17].

\subsection{Uniqueness of the NEP}

Having settled the question of existence of a NEP, we now establish its uniqueness under appropriate conditions. Let us first introduce the gradient column vector:

$$
g_{l}(f)=\left[\nabla_{1} F_{l}^{u, 1}\left(f_{l}^{1}, f_{l}\right), \ldots, \nabla_{I} F_{l}^{u, I}\left(f_{l}^{I}, f_{l}\right)\right]^{T}
$$

where $\nabla_{i} F_{l}^{u, i}\left(f_{l}^{i}, f_{l}\right)$ denotes the gradient of $F_{l}^{u, i}\left(f_{l}^{i}, f_{l}\right)$ with respect to $f_{l}^{i}$ and $f_{l}:=\left\{f_{l}^{i} ; i=1, \ldots, I\right\}$. Then, it follows from Theorem 2 of [17], in view of Corollary 3.1 of [1] (which translates Rosen's diagonal strict concavity condition to a similar condition applied to individual links), that the NEP is unique if for any set of vectors $f_{l}$ and $\tilde{f}_{l}, l \in \mathcal{L},\left(f_{l} \neq \tilde{f}_{l} \forall l \in\right.$ $\mathcal{L}$, in the vector sense), satisfying the flow constraints, we have:

$$
\sum_{l \in \mathcal{L}}\left(f_{l}-\tilde{f}_{l}\right)^{T} \cdot\left(g_{l}\left(f_{l}\right)-g_{l}\left(\tilde{f}_{l}\right)\right)<0
$$

We will show that in fact, for any $l$ for which $f_{l} \neq \tilde{f}_{l}$, every term in the above summation is negative, i.e.,

$$
\left(f_{l}-\tilde{f}_{l}\right)^{T} \cdot\left(g_{l}\left(f_{l}\right)-g_{l}\left(\tilde{f}_{l}\right)\right)<0
$$

We first consider the case $\beta(l)=1$, for which the expression in $(10)$ is quadratic in $\left(f_{l}-\tilde{f}_{l}\right)$. Then, all we need to show is that the Jacobian, $G_{l}\left(f_{l}\right)$, of $g_{l}\left(f_{l}\right)$ with respect to $f_{l}$ is negative-definite. Simple calculation yields:

$$
G_{l}\left(f_{l}\right)=\left\{\frac{\partial^{2} F_{l}^{u, i}\left(f_{l}^{i}, f_{l}\right)}{\partial f_{l}^{i} \partial f_{l}^{j}}\right\}_{i, j}=-\left(\underline{11}^{T}+M\right) \cdot a_{l},
$$

where 1 denotes the $I$-dimensional vector with entries all equal to 1 , and $M$ is the identity matrix. $G_{l}$ is clearly negative-definite, and hence the negativity condition (10) holds. Therefore, the Nash equilibrium point is unique (for this special case of $\beta(l)=1$ ).

Next, we consider the case of a general $\beta(l)$. The Jacobian of $g_{l}\left(f_{l}\right)$ is: 


$$
G_{l}\left(f_{l}\right)=-a_{l} \cdot \beta(l) \cdot\left(f_{l}\right)^{\beta(l)-2} \cdot\left(q_{l} \cdot \underline{1}^{T}+f_{l} \cdot M\right),
$$

where $q_{l}$ is an $I$-dimensional vector whose $i$-th entry is $q_{l}^{i}=f_{l}+(\beta(l)-1) \cdot f_{l}^{i}$.

It follows from Theorem 6 of [17], brought to the link level in view of Corollary 3.1 of [1], that a sufficient condition for (10) is for the symmetric matrix $G_{l}\left(f_{l}\right)+G_{l}\left(f_{l}\right)^{T}$ to be negative-definite for all nonnegative $f_{l}$ value.

A careful look at the proof of Theorem 6 of [17] will actually reveal that it is sufficient to require negative definiteness for all but isolated values of $f_{l}$, and in particular for all $f_{l}$ such that the total flow over that link is positive (that is, at least one of the components of $f_{l}$ is positive).

In accordance with this, it will be sufficient to show that for $f_{l}>0$, the eigenvalues of $-\left[\left(q_{l} \cdot \underline{1}^{T}+f_{l} M\right)+\left(q_{l} \cdot \underline{1}^{T}+f_{l} M\right)^{T}\right]$ are all negative, or equivalently $\bar{A}_{l}:=q_{l} \cdot \underline{1}^{T}+\underline{1} \cdot q_{l}^{\bar{T}}>-2 f_{l} M$.

It can be easily demonstrated that the eigenvalues of $A_{l}$ are $z+r-2 f_{l}$ and $z-r-2 f_{l}$, where $z:=\underline{1}^{T} \cdot q_{l}+2 f_{l}=$ $(I+1+\beta(l)) f_{l}$ and $r:=\sqrt{I q_{l}^{T} q_{l}}$, and they are both greater than $-2 f_{l}$. Therefore, the NEP is indeed unique. The following Section is dedicated to computing the Nash equilibrium point.

\section{COMPUTING THE SOLUTION}

For a given price vector $p=\left(p_{1}, p_{2}, \ldots, p_{L}\right) \in R^{L}$, we have a (non-cooperative) routing game between the users. Each user $i$ aims at maximizing his objective function $F^{u, i}$, which has the following expression, considering the cost function illustrated in Section 3.3:

$F^{u, i}\left(f^{i}, f^{-i}, p\right)=\sum_{l \in \mathcal{L}} \alpha_{l}^{i} \cdot f_{l}^{i}-\sum_{l \in \mathcal{L}} f_{l}^{i} \cdot\left[a_{l} \cdot\left(f_{l}\right)^{\beta(l)}+b_{l}+p_{l}\right]$

The optimal flows of the users can be obtained by solving the set of first-order conditions: for $i \in \mathcal{I}$,

$$
\left(\partial / \partial f_{l}^{i}\right) F^{u, i} \begin{cases}=0 & , \text { if } f_{l}^{i}>0 \\ <0 & , \text { if } f_{l}^{i}=0\end{cases}
$$

which yields the following expression of the $i-$ th user flow on link $l$ :

$$
f_{l}^{i}\left(p_{l}\right)=\frac{\alpha_{l}^{i}-\left[a_{l}\left(f_{l}\right)^{\beta(l)}+b_{l}+p_{l}\right]}{a_{l} \beta(l)\left(f_{l}\right)^{[\beta(l)-1]}},
$$

If we sum over all $i \in \mathcal{I}$ we obtain:

$$
f_{l}\left(p_{l}\right)=\left[\frac{\sum_{i \in \mathcal{I}} \alpha_{l}^{i}-I \cdot\left(b_{l}+p_{l}\right)}{(I+\beta(l)) a_{l}}\right]^{1 / \beta(l)}
$$

and the condition $f_{l}>0$ implies that $p_{l}<\hat{p}=\sum_{i \in \mathcal{I}} \alpha_{l}^{i} / I-$ $b_{l}$.

We can now express $p_{l}$ in function of $f_{l}$ :

$$
p_{l}\left(f_{l}\right)=\sum_{i \in \mathcal{I}} \alpha_{l}^{i} / I-b_{l}-(1+\beta(l) / I) \cdot a_{l} \cdot\left(f_{l}\right)^{\beta(l)}
$$

At this stage, Service Provider $l$ should solve the problem of maximizing the objective function $p_{l}\left(f_{l}\right) \cdot f_{l}=f_{l}$. $\left[\sum_{i \in \mathcal{I}} \alpha_{l}^{i} / I-b_{l}-(1+\beta(l) / I) \cdot a_{l} \cdot\left(f_{l}\right)^{\beta(l)}\right]$ with respect to $f_{l}$.

The solution is:

$$
f_{l}^{*}=\left[\frac{\sum_{i \in \mathcal{I}} \alpha_{l}^{i}-I \cdot b_{l}}{a_{l} \cdot(1+\beta(l)) \cdot(I+\beta(l))}\right]^{1 / \beta(l)}
$$

provided that $\sum_{i \in \mathcal{I}} \alpha_{l}^{i} / I>b_{l}$, which ensures that $f_{l}^{*}>0$.

The optimal value of $p_{l}$ is:

$$
p_{l}^{*}=\frac{\beta(l)}{1+\beta(l)} \cdot\left[\sum_{i \in \mathcal{I}} \alpha_{l}^{i} / I-b_{l}\right]
$$

and it can be easily checked to be positive provided that $\sum_{i \in \mathcal{I}} \alpha_{l}^{i} / I>b_{l}$, a condition already assumed to hold. It is also easy to check that $p_{l}^{*}<\hat{p}$.

The optimal values of $f_{l}^{i}$ are:

$$
f_{l}^{i *}=\frac{\mathrm{A}}{\mathrm{B}}
$$

where A and B have the following expressions:

$$
\begin{array}{r}
\mathrm{A}=\alpha_{l}^{i} I(1+\beta(l))(I+\beta(l))- \\
-\left(\sum_{i \in \mathcal{I}} \alpha_{l}^{i}\right)\left[I+I \beta(l)+\beta(l)^{2}\right]-I \beta(l) b_{l} \\
\mathrm{~B}=I \beta(l)\left[a_{l}(1+\beta(l))(I+\beta(l))\right]^{1 / \beta(l)} \\
\cdot\left[\sum_{i \in \mathcal{I}} \alpha_{l}^{i}-I b_{l}\right]^{(1-1 / \beta(l))}
\end{array}
$$

Now we must derive the conditions under which the individual flows are positive. It is easy to check that the denominator is positive provided that $\sum_{i \in \mathcal{I}} \alpha_{l}^{i} / I>b_{l}$. So $f_{l}^{i *}>$ $0 \Rightarrow\left(I+I \beta(l)+\beta(l)^{2}\right)\left(\alpha_{l}^{i}-\sum_{i \in \mathcal{I}} \alpha_{l}^{i} / I\right)+\beta(l) \alpha_{l}^{i}>\beta(l) b_{l}$. 
For $\beta(l)=1$,

$$
f_{l}^{i *}=\frac{1}{a_{l}} \cdot\left[\alpha_{l}^{i}-\frac{(1+2 I)}{2 I(1+I)} \cdot\left(\sum_{i \in \mathcal{I}} \alpha_{l}^{i}\right)-\frac{b_{l}}{2(1+I)}\right]
$$

and $f_{l}^{i *}>0 \Rightarrow \alpha_{l}^{i}>\frac{(1+2 I) \cdot\left(\sum_{i \in \mathcal{I}} \alpha_{l}^{i} / I\right)+b_{l}}{2(I+1)}$.

Note that, for $\beta(l)=1$, the condition $\sum_{i \in \mathcal{I}} \alpha_{l}^{i} / I>b_{l}$ implies that $\frac{(1+2 I) \cdot\left(\sum_{i \in \mathcal{I}} \alpha_{l}^{i} / I\right)+b_{l}}{2(I+1)}>b_{l}$, and from the previous expression $\left(\alpha_{l}^{i}>\frac{(1+2 I) \cdot\left(\sum_{i \in \mathcal{I}} \alpha_{l}^{i} / I\right)+b_{l}}{2(I+1)}\right)$, we can write the following: $\alpha_{l}^{i}>\frac{(1+2 I) \cdot\left(\sum_{i \in \mathcal{I}} \alpha_{l}^{i} / I\right)+b_{l}}{2(I+1)}>b_{l}$.

\section{SOLUTION FOR A SPECIAL CASE: SYM- METRICAL USERS}

We consider here a special case of the problem formulated above, where all users have the same utility per unit of flow on link $l$, i.e., $\alpha_{l}^{i}=\alpha_{l}, \forall i \in \mathcal{I}, l \in \mathcal{L}$, and as a consequence they share the same objective function.

This special case permits to derive simpler equilibrium expressions, providing further insights into the model solution.

Lemma 1: In the considered parallel links network with symmetrical users, the price and flow values at the Nash Equilibrium Point are such that

$$
p_{l}^{*}=\frac{\beta(l)}{1+\beta(l)} \cdot\left(\alpha_{l}-b_{l}\right)
$$

and

$$
f_{l}^{i *}=\frac{f_{l}^{*}}{I}
$$

where

$$
f_{l}^{*}=\left[\frac{I\left(\alpha_{l}-b_{l}\right)}{a_{l}(1+\beta(l))(I+\beta(l))}\right]^{1 / \beta(l)}
$$

Furthermore, for $\beta(l)=1, p_{l}^{*}$ and $f_{l}^{*}$ become:

$$
p_{l}^{*}=\frac{\alpha_{l}-b_{l}}{2}
$$

and

$$
f_{l}^{*}=\frac{I\left(\alpha_{l}-b_{l}\right)}{2 a_{l}(I+1)}
$$

Proof: The above expressions of the equilibrium prices and flows can be easily derived following the same procedure used in Section 5 for computing the general solution.

\section{EXTENDED MODEL WITH ACCESS COSTS}

We now extend the joint routing and pricing problem by considering access costs in addition to transport costs. We assume that such costs are both user and Provider-dependent, i.e., they are related to the coverage deployed by each Service Provider. This is in line, for example, with the access service provided by local ISPs that compete for different sets of users.

To this aim, we reformulate the problem as follows: each user $i$ wants to connect two remote end-points (e.g., two different sites or offices), referred to as $s_{i}$ and $t_{i}$. To this aim, $L$ Service Providers (SPs) are available. Each user can split his traffic over multiple SPs; furthermore, the access links connecting $s_{i}$ and $t_{i}$ to the SPs' link are characterized by an access cost that we denote by $A C s_{l}^{i}$ and $A C t_{l}^{i}$, respectively. Figure 2 illustrates the network scenario we are considering.

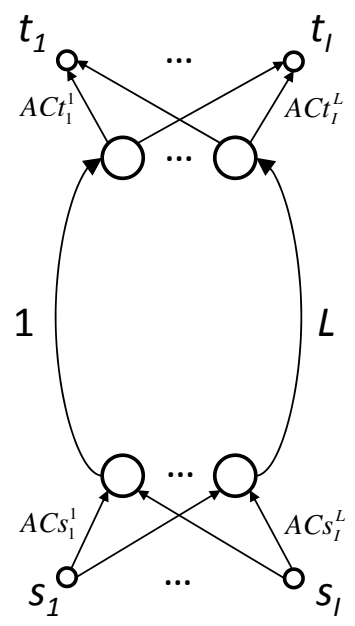

Figure 2: Extended Model with access costs: each user wants to connect two remote end-points, $s_{i}$ and $t_{i}$, using the transfer services offered by $L$ different Service Providers. Users can split their traffic, and face both access costs $\left(A C s_{l}^{i}\right.$ and $\left.A C t_{l}^{i}\right)$ and transport costs $\left(p_{l}\right)$.

This problem can be modeled extending the parallel links network model considered in Section 5, taking into account the access costs between the $I$ end-users and the $L$ Service Providers.

In this model, we assume a usage-based access pricing: access costs $A C s_{l}^{i}$ and $A C t_{l}^{i}$ represent per bandwidth unit costs, and therefore the total access cost for user $i$ is equal to $\sum_{l \in \mathcal{L}} f_{l}^{i} \cdot\left(A C s_{l}^{i}+A C t_{l}^{i}\right)$.

The user $i$ 's objective function becomes as follows:

$$
\begin{array}{r}
F_{A C}^{u, i}\left(f^{i}, f^{-i}, p\right)=\sum_{l \in \mathcal{L}} \alpha_{l}^{i} \cdot f_{l}^{i}- \\
-\sum_{l \in \mathcal{L}}\left(f_{l}^{i} \cdot\left[a_{l} \cdot\left(f_{l}\right)^{\beta(l)}+A C s_{l}^{i}+A C t_{l}^{i}+b_{l}+p_{l}\right]\right)
\end{array}
$$


It is easy to observe that the existence and uniqueness conditions derived for the previous basic model still hold for the extended model.

Hereafter, we use the same procedure considered in Section 5 to compute the Nash equilibrium, including the access costs in the expressions of the optimal users' flows and prices.

\section{Computing the Solution for the Extended Model with Access Costs}

The optimal flows of the users can be obtained by solving the set of first-order conditions, for $i \in \mathcal{I}$,

$$
\left(\partial / \partial f_{l}^{i}\right) F_{A C}^{u, i} \begin{cases}=0 & , \text { if } f_{l}^{i}>0 \\ <0 & , \text { if } f_{l}^{i}=0\end{cases}
$$

which leads to the following expression for the $i$-th user flow on link $l$ :

$$
f_{l}^{i}\left(p_{l}\right)=\frac{\alpha_{l}^{i}-\left[a_{l}\left(f_{l}\right)^{\beta(l)}+A C s_{l}^{i}+A C t_{l}^{i}+b_{l}+p_{l}\right]}{a_{l} \beta(l)\left(f_{l}\right)^{[\beta(l)-1]}}
$$

If we sum over all $i \in \mathcal{I}$ we obtain:

$f_{l}=\left[\frac{\sum_{i \in \mathcal{I}} \alpha_{l}^{i}-\sum_{i \in \mathcal{I}}\left(A C s_{l}^{i}+A C t_{l}^{i}\right)-I \cdot\left(b_{l}+p_{l}\right)}{(I+\beta(l)) a_{l}}\right]^{1 / \beta(l)}$

$f_{l}>0 \Rightarrow p_{l}<\hat{p}=\sum_{i \in \mathcal{I}} \alpha_{l}^{i} / I-\sum_{i \in \mathcal{I}}\left(A C s_{l}^{i}+A C t_{l}^{i}\right) / I-b_{l}$

We can now express $p_{l}$ in function of $f_{l}$ :

$$
\begin{array}{r}
p_{l}=\sum_{i \in \mathcal{I}} \alpha_{l}^{i} / I-\sum_{i \in \mathcal{I}}\left(A C s_{l}^{i}+A C t_{l}^{i}\right) / I-b_{l}- \\
-(1+\beta(l) / I) \cdot a_{l} \cdot\left(f_{l}\right)^{\beta(l)}
\end{array}
$$

Service Provider $l$ should solve the problem of maximizing the objective function $p_{l} \cdot f_{l}=f_{l} \cdot\left[\sum_{i \in \mathcal{I}} \alpha_{l}^{i} / I-\sum_{i \in \mathcal{I}}\left(A C s_{l}^{i}+\right.\right.$ $\left.\left.A C t_{l}^{i}\right) / I-b_{l}-(1+\beta(l) / I) \cdot a_{l} \cdot\left(f_{l}\right)^{\beta(l)}\right]$ with respect to $f_{l}$.

The solution for the total flow traversing link $l$ is:

$$
f_{l}^{*}=\left[\frac{\sum_{i \in \mathcal{I}} \alpha_{l}^{i}-\sum_{i \in \mathcal{I}}\left(A C s_{l}^{i}+A C t_{l}^{i}\right)-I \cdot b_{l}}{a_{l} \cdot(1+\beta(l)) \cdot(I+\beta(l))}\right]^{1 / \beta(l)}
$$

provided that $\sum_{i \in \mathcal{I}} \alpha_{l}^{i}>\sum_{i \in \mathcal{I}}\left(A C s_{l}^{i}+A C t_{l}^{i}\right)+I \cdot b_{l}$; this last condition ensures that $f_{l}^{*}>0$.

The optimal value of $p_{l}$ is:

$$
p_{l}^{*}=\frac{\beta(l)}{I(1+\beta(l))} \cdot\left[\sum_{i \in \mathcal{I}} \alpha_{l}^{i}-\sum_{i \in \mathcal{I}}\left(A C s_{l}^{i}+A C t_{l}^{i}\right)-I \cdot b_{l}\right]
$$

and it is positive provided that $\sum_{i \in \mathcal{I}} \alpha_{l}^{i}>\sum_{i \in \mathcal{I}}\left(A C s_{l}^{i}+\right.$ $\left.A C t_{l}^{i}\right)+I \cdot b_{l}$, a condition already assumed to hold. It is also easy to check that $p_{l}^{*}<\hat{p}$.

Hence, the optimal values of $f_{l}^{i}$ are:

$$
f_{l}^{i *}=\frac{\mathrm{C}}{\mathrm{D}}
$$

where $\mathrm{C}$ and $\mathrm{D}$ have the following expressions:

$$
\begin{array}{r}
\mathrm{C}=\left(\alpha_{l}^{i}-A C s_{l}^{i}-A C t_{l}^{i}\right)[I(1+\beta(l))(I+\beta(l))]- \\
-\left(\sum_{i \in \mathcal{I}} \alpha_{l}^{i}-\sum_{i \in \mathcal{I}}\left(A C s_{l}^{i}+A C t_{l}^{i}\right)\right)\left[I+I \beta(l)+(\beta(l))^{2}\right]- \\
-I \beta(l) b_{l}
\end{array}
$$

$$
\begin{array}{r}
\mathrm{D}=I \beta(l)\left[a_{l}(1+\beta(l))(I+\beta(l))\right]^{1 / \beta(l)} . \\
\cdot\left[\sum_{i \in \mathcal{I}} \alpha_{l}^{i}-\sum_{i \in \mathcal{I}}\left(A C s_{l}^{i}+A C t_{l}^{i}\right)-I b_{l}\right]^{(1-1 / \beta(l))}
\end{array}
$$

The individual flows are positive under the following condition: $\left(I+I \beta(l)+\beta(l)^{2}\right)\left[\left(\alpha_{l}^{i}-\sum_{i \in \mathcal{I}} \alpha_{l}^{i} / I\right)-\left(A C s_{l}^{i}+A C t_{l}^{i}-\right.\right.$ $\left.\left.\sum_{i \in \mathcal{I}}\left(A C s_{l}^{i}+A C t_{l}^{i}\right) / I\right)\right]+\beta(l)\left(\alpha_{l}^{i}-A C s_{l}^{i}-A C t_{l}^{i}\right)>\beta(l) b_{l}$.

For $\beta(l)=1$,

$$
\begin{aligned}
& f_{l}^{i *}=\frac{1}{a_{l}}\left(\alpha_{l}^{i}-A C s_{l}^{i}-A C t_{l}^{i}\right)-\frac{b_{l}}{2 a_{l}(1+I)}- \\
& -\frac{(1+2 I)}{2 a_{l} I(1+I)}\left(\sum_{i \in \mathcal{I}} \alpha_{l}^{i}-\sum_{i \in \mathcal{I}}\left(A C s_{l}^{i}+A C t_{l}^{i}\right)\right)
\end{aligned}
$$

and $f_{l}^{i *}>0 \Rightarrow \alpha_{l}^{i}>A C s_{l}^{i}+A C t_{l}^{i}+$ $+\frac{(1+2 I) \cdot\left(\sum_{i \in \mathcal{I}} \alpha_{l}^{i}-\sum_{i \in \mathcal{I}}\left(A C s_{l}^{i}+A C t_{l}^{i}\right)\right)+I \cdot b_{l}}{2 I(I+1)}$.

\section{NUMERICAL RESULTS}

In this Section, we analyze and discuss the numerical results obtained solving our proposed models. We first consider the basic model, testing the sensitivity of the solution to different $\alpha_{l}^{i}$ settings. Then, we measure the Service Providers' revenue as the number of network users increases. Finally, we consider the extended model that takes into account users' access costs, measuring their impact on the Service Providers' revenue.

We first consider a network scenario with only 2 identical users that share a single link $l$ (i.e., $L=1$ ), owned by a 
single Service Provider. The parameters values are set in such a way that the conditions derived while computing the solution of the basic model in Section 5 are satisfied: $\alpha_{l}^{i}=$ $1, \forall i \in \mathcal{I}, l \in \mathcal{L}, a_{l}=1, b_{l}=0.5$ and $\beta(l)=1, \forall l \in \mathcal{L}$.

The Nash Equilibrium solution is therefore $p_{l}^{*}=1 / 4$ and $f_{l}^{1 *}=f_{l}^{2 *}=1 / 12$.

Figure 3 illustrates the Service Provider utility function in this scenario, $U_{l}^{S P}=p_{l} \cdot f_{l}$, as a function of the price $p_{l}$. The SP's revenue evidently exhibits a maximum for $p_{l}=1 / 4$.

Figure 4, on the other hand, shows the user objective function (which is identical for both users) $F_{l}^{u, i}=\alpha_{l}^{i} \cdot f_{l}^{i}-f_{l}^{i}$. $\left[a_{l} \cdot\left(f_{l}\right)^{\beta(l)}+b_{l}+p_{l}\right]$ as a function of the users' flow values $\left(f_{l}^{i}\right)$. In this Figure, the price $p_{l}$ was set equal to $1 / 4$, so that the Service Provider maximizes its revenue.

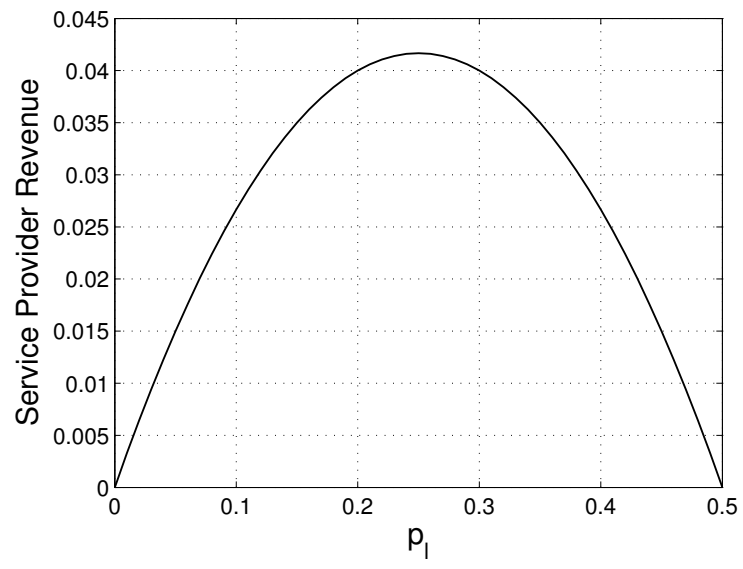

Figure 3: Service Provider revenue as a function of the price $p_{l}$ set on link $l$.

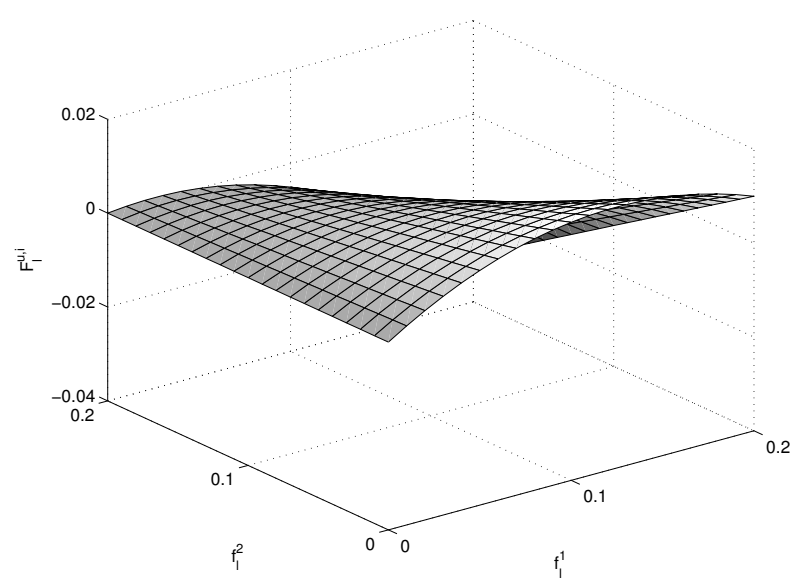

Figure 4: User objective function in the two-user network scenario as a function of $f_{l}^{1}$ and $f_{l}^{2}$, with $p_{l}=p_{l}^{*}=1 / 4$.

We then measured the amount of flow routed into the net- work by users having different $\alpha_{l}^{i}$ values, i.e., different per bandwidth unit utilities. To this aim, we considered a scenario with $I=10$ users, 5 of which having $\alpha_{l}^{i}=4+\epsilon$, the other 5 having $\alpha_{l}^{i}=4-\epsilon$, with $\epsilon$ in the 0 to 0.1 range. In this way, since $\sum_{i \in I} \alpha_{l}^{i}=40=$ constant, the total flow on each link $l$ is always the same for each $\epsilon$ value (see expression (14)). The other parameters, $a_{l}, b_{l}$ and $\beta(l)$, are set as in the previous scenario.

Figure 5(a) reports the equilibrium flow $\left(f_{l}^{i *}\right)$ routed over link $l$ by two users belonging to each one of the two groups, as a function of $\epsilon$. Figure 5(b) illustrates the ratio between the flow routed by users having $\alpha_{l}^{i}=4+\epsilon$ and those having $\alpha_{l}^{i}=4-\epsilon$

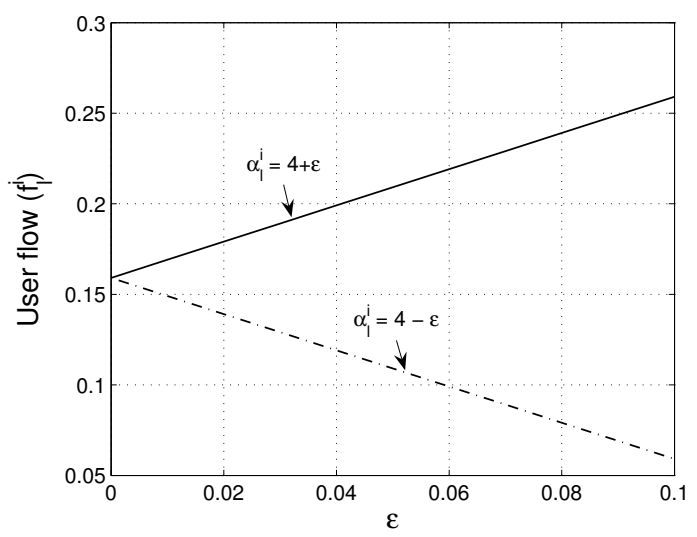

(a)

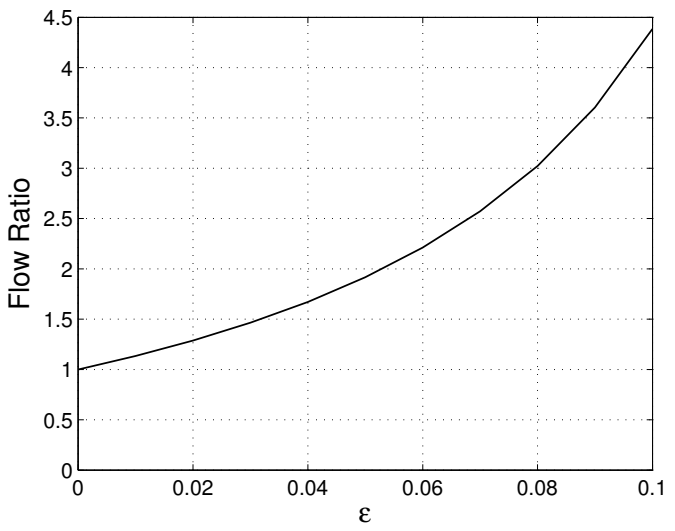

(b)

Figure 5: Network scenario with $I=10$ users, 5 of which having $\alpha_{l}^{i}=4+\epsilon$, the other 5 having $\alpha_{l}^{i}=4-\epsilon$ : (a) equilibrium flow sent by each user on link $l$ (b) ratio between the flow routed by users having $\alpha_{l}^{i}=$ $4+\epsilon$ and those having $\alpha_{l}^{i}=4-\epsilon$.

It can be observed that the amount of flow $f_{l}^{i *}$ routed by 
each user consistently increases with increasing $\alpha_{l}^{i}$ values, so that even small increases in $\epsilon$ lead to quite large differences in $f_{l}^{i *}$.

We then measured the revenue achieved by a Service Provider as a function of the number of network users $I$, considering a scenario with a single link, $\alpha_{l}^{i}=4, a_{l}=1, b_{l}=0.5$ and $\beta(l)=1$. Figure 6 illustrates the SP's revenue at the Nash Equilibrium point, $p_{l}^{*} f_{l}^{*}$, as a function of $I$. For simplicity, we consider the symmetrical users case studied in Section 6 . The equilibrium price, $p_{l}^{*}$, does not depend from the users' number, since it is equal to $p_{l}^{*}=\frac{\alpha_{l}-b_{l}}{2}$, while the equilibrium flow on link $l, f_{l}^{*}$, increases with increasing $I$ values, $f_{l}^{*}=\frac{I\left(\alpha_{l}-b_{l}\right)}{2 a_{l}(I+1)}$. Hence, the revenue achieved by the $l$-th Service Provider increases with increasing $I$ values, and is upper bounded by $\frac{\left(\alpha_{l}-b_{l}\right)^{2}}{4 a_{l}}$ (which is equal to 3.0625 in this scenario).

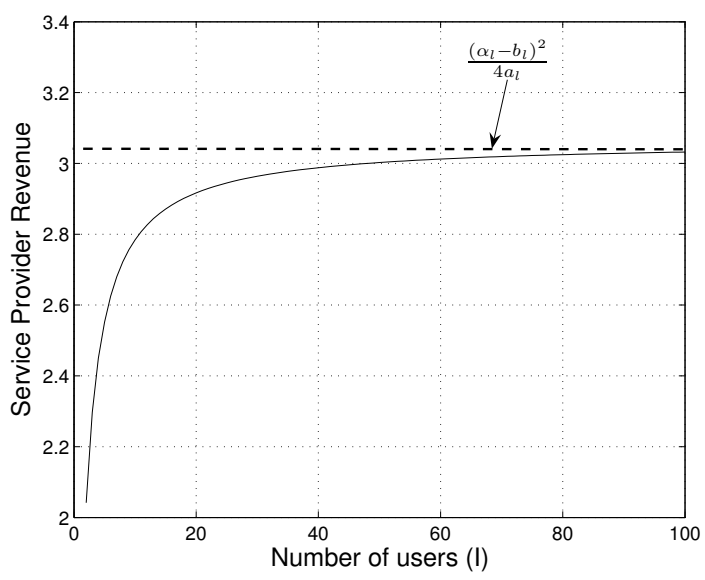

Figure 6: Service Provider revenue as a function of the number of users, I (Symmetrical users case).

Finally, in the same scenario we considered the extended model with access costs, measuring the revenue obtained by Service Providers as a function of the network users' number, $I$, under various access cost settings. Figure 7 illustrates the results with different $A C s_{l}^{i}$ and $A C t_{l}^{i}$ values, namely $A C s_{l}^{i}=A C t_{l}^{i}=1$ and 2 . For comparison reasons we also reported the $A C s_{l}^{i}=A C t_{l}^{i}=0$ case, which was already illustrated in Figure 6.

It can be observed that, as the access costs increase, the SP revenue decreases; this behavior is more evident especially when the number of users is small (i.e., for $I<20$ ).

\section{CONCLUSION}

In this paper we studied the economic interaction between network users and Service Providers. Users are characterized by elastic traffic, and face access and transport cost. Service Providers compete among themselves to cover network users and set transport prices to maximize their revenue.

We modeled this problem with a two-stage (Stackelberg) game, where Service Providers set prices per bandwidth unit

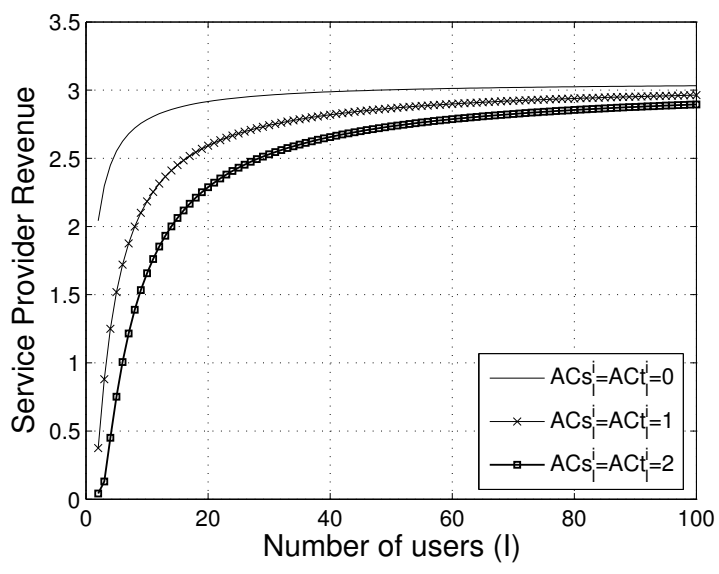

Figure 7: Service Provider revenue as a function of the number of users $I$ and access costs, (Symmetrical users case).

and users respond by presenting a certain amount of flow to the network.

We demonstrated the existence and uniqueness of the Nash equilibrium considering both a basic and an extended model that takes into account the users' access costs. Finally, we illustrated several numerical examples that provide insights into the model solution.

\section{Acknowledgments}

This work was partially supported by MIUR in the framework of the PRIN SESAME project.

\section{REFERENCES}

[1] A. Orda, R. Rom, and N. Shimkin. Competitive routing in multiuser communication networks. IEEE/ACM Transactions on Networking, pages 510-521, vol. 1(5), 1993.

[2] Y.A. Korilis, A.A. Lazar, and A. Orda. Architecting noncooperative networks. IEEE Journal on Selected Areas in Communications, pages 1241-1251, vol. 13, no. 7, 1995.

[3] E. Altman, T. Basar, T. Jimenez, and N. Shimkin. Competitive routing in networks with polynomial costs. IEEE Transactions on Automatic Control, pages 92-96, vol. 47(1), 2002.

[4] S. Dafermos and F. Sparrow. The traffic assignment problem for a general network. J. Res. Natl. Bur. Stand., Sect. B, pages 91-118, vol. 73, 1969.

[5] A. Hayrapetyan, E. Tardos, and T. Wexler. A network pricing game with selfish traffic. Distributed Computing, pages 255-266, vol. 19(4), March 2007.

[6] A. Ozdaglar. Price competition with elastic traffic. Networks, pages 141-155, vol. 52(3), October 2008.

[7] D. Acemoglu and A. Ozdaglar. Flow control, routing, and performance from service provider viewpoint. LIDS report WP1696, December 2003.

[8] T. Basar and R. Srikant. Revenue-maximizing pricing and capacity expansion in a many-users regime. 
Infocom 2002, pages 294-301, vol. 1, 2002.

[9] T. Basar and R. Srikant. A Stackelberg network game with a large number of followers. Journal of Optimization Theory and Applications, pages 479-490, vol. 115(3), December 2002.

[10] T. Basar and G. J. Olsder. Dynamic noncooperative game theory. SIAM Series in Classics in Applied Mathematics, Philadelphia, PA, 1999.

[11] Z. Duan, Z.-L. Zhang, and Y.T. Hou. Service overlay networks: SLAs, QoS, and bandwidth provisioning. IEEE/ACM Transactions on Networking, pages 870-883, vol. 11, no. 6, December 2003.

[12] Z. Li and P. Mohapatra. QRON: QoS-aware routing in overlay networks. IEEE Journal on Selected Areas in Communications, pages 29-40, vol. 22, no. 1, January 2004.

[13] H.T. Tran and T. Ziegler. A design framework towards the profitable operation of service overlay networks. Computer Networks, pages 94-113, vol. 51, 2007.

[14] G. Armitage. MPLS: the magic behind the myths [multiprotocol label switching]. IEEE Communications Magazine, pages 124-131, vol. 38, no. 1, 2000.

[15] D. Acemoglu and A. Ozdaglar. Competition and efficiency in congested markets. Mathematics of Operations Research, pages 1-32, vol. 32(1), February 2007.

[16] O.F.P.R. BUREAU. Traffic Assignment Manual. US Department of Commerce, Washington, DC, 1964.

[17] J.B. Rosen. Existence and uniqueness of equilibrium points for concave n-person games. Econometrica, pages 520-534, vol. 33(3), 1965. 